TRANSACTIONS OF THE

AMERICAN MATHEMATICAL SOCIETY

Volume 363, Number 10, October 2011, Pages 5465-5480

S 0002-9947(2011)05306-7

Article electronically published on April 27, 2011

\title{
KOLMOGOROV COMPLEXITY AND THE RECURSION THEOREM
}

\author{
BJØRN KJOS-HANSSEN, WOLFGANG MERKLE, AND FRANK STEPHAN
}

\begin{abstract}
Several classes of diagonally nonrecursive (DNR) functions are characterized in terms of Kolmogorov complexity. In particular, a set of natural numbers $A$ can wtt-compute a DNR function iff there is a nontrivial recursive lower bound on the Kolmogorov complexity of the initial segments of $A$. Furthermore, $A$ can Turing compute a DNR function iff there is a nontrivial $A$-recursive lower bound on the Kolmogorov complexity of the initial segments of $A$. $A$ is PA-complete, that is, $A$ can compute a $\{0,1\}$-valued DNR function, iff $A$ can compute a function $F$ such that $F(n)$ is a string of length $n$ and maximal $C$-complexity among the strings of length $n . A \geq_{T} K$ iff $A$ can compute a function $F$ such that $F(n)$ is a string of length $n$ and maximal $H$-complexity among the strings of length $n$. Further characterizations for these classes are given. The existence of a DNR function in a Turing degree is equivalent to the failure of the Recursion Theorem for this degree; thus the provided results characterize those Turing degrees in terms of Kolmogorov complexity which no longer permit the usage of the Recursion Theorem.
\end{abstract}

\section{INTRODUCTION}

The Recursion Theorem can be stated in two ways: First one can say that every recursive function $f$ has a fixed point with respect to any given acceptable numbering of all r.e. sets: $\exists e\left[W_{e}=W_{f(e)}\right]$. Second one can say that every total recursive function $f$ coincides at some places with the diagonal function: $\exists e\left[\varphi_{e}(e) \downarrow\right.$ $=f(e)]$. Jockusch [5] showed that these two variants of the Recursion Theorem are also equivalent relative to any oracle $A$ : Every function $f \leq_{T} A$ admits a fixed point iff every function $g \leq_{T} A$ coincides with the diagonal function somewhere. Special attention has been given to the oracles $A$ which permit us to avoid the Recursion Theorem, and the topic of the present work is to relate these oracles to notions of Kolmogorov complexity, namely to the classes of complex and autocomplex sets introduced below where one can give a nontrivial lower bound on the complexity of the initial segments of the set observed. The formal definitions of fixed-point free and diagonally nonrecursive functions are as follows.

Definition 1.1. A function $f$ is called fixed-point free if $W_{x} \neq W_{f(x)}$ for all $x$, where $W_{x}$ is the $x$ th recursively enumerable set. The partial recursive function mapping $x$ to $\varphi_{x}(x)$ whenever defined is called the diagonal function, where $\varphi_{x}$ is the $x$ th partial recursive function. A function $g$ is called diagonally nonrecursive (DNR) iff it is total and differs from the diagonal function on its domain.

Received by the editors December 23, 2009 and, in revised form, February 2, 2010.

2010 Mathematics Subject Classification. Primary 03D28, 03D32, 68Q30.

The first author was partially supported by NSF-USA grants DMS-0652669 and DMS-0901020. 
We also relate complexity to sparseness: Suppose sets $A$ and $B$ are given such that $A \leq_{w t t} B$ and $B$ is hyperimmune. Then $A$ is computed by querying rather few bits from $B$, and $B$ has very long intervals consisting of zeroes only. So when computing $A(x)$ for many inputs $x$, the oracle $B$ will answer " 0 " on all questions that have not been asked before; hence $B$ will not be of much use. We show that this can happen for some $B$ just in case $A$ has low Kolmogorov complexity in a certain sense. This is again equivalent to the statement that the Recursion Theorem applies to recursively bounded functions wtt-reducible to $A$.

A further topic investigated is to determine how difficult it is to compute for each $n$ a string of maximal Kolmogorov complexity within $\{0,1\}^{n}$. While for the case of plain Kolmogorov complexity the answer to this problem depends on the universal machine under consideration, in the case of prefix-free Kolmogorov complexity the answer is that the problem to compute such strings is as difficult to solve as the halting problem.

Besides this we study related questions and are able to characterize the oracles which are high or autocomplex; furthermore, related characterizations are obtained for the oracles which are high or PA-complete. In the last section we study the notion of r.e. traceable sets which are, roughly speaking, some type of notion opposite the notion of autocomplex sets.

\section{Complex sets}

A set will be called complex if the prefixes of its characteristic function have a nontrivial lower bound computed by some recursive function; this notion is a weakening of the corresponding characterization for randomness where this lower bound is just the length of the prefix minus some constant. Recall that $C$ is the plain and $H$ the prefix-free Kolmogorov complexity.

Recall that a function $g: \omega \rightarrow \omega$ is called an order function if it is recursive, nondecreasing, and unbounded. For a set $A$, we say that the function $g: \omega \rightarrow \omega$ is an $A$-order function if it is recursive in $A$, nondecreasing, and unbounded.

Definition 2.1. A set $A$ is complex if there is an order function $g$ such that $C(A \uparrow y) \geq g(y)$ for all $y$.

A set $A$ is autocomplex if there is an $A$-order function $g$ such that $C(A\lceil y) \geq g(y)$ for all $y$.

Here $A \uparrow x$ is the finite binary sequence $A(0) \ldots A(x-1)$.

Proposition 2.2. For any set $A$, the following conditions are equivalent.

(1) The set $A$ is autocomplex.

(2) There is an A-recursive function $h$ such that for all $n, C(A \uparrow h(n)) \geq n$.

(3) There is an A-recursive function $f$ such that for all $n, C(f(n)) \geq n$.

Proof. We show $(1) \Rightarrow(2) \Rightarrow(3) \Rightarrow(1)$. Given an autocomplex set $A$, choose an $A$-recursive order $g$, where $C(A \uparrow n) \geq g(n)$, and in order to obtain a function $h$ as required by (2), let

$$
h(n)=\min \{l: g(l) \geq n\} .
$$

Given a function $h$ as in (2), in order to obtain a function $f$ as required by (3), simply let $f(n)$ be equal to (an appropriate encoding of) the prefix of $A$ of length $h(n)$. Finally, given an $A$-recursive function $f$ as in (3), let $u(n)$ be an $A$-recursive order such that some fixed oracle Turing machine $M$ computes $f$ with oracle $A$ such 
that $M$ queries on input $n$ only bits $A(m)$ of $A$ where $m \leq u(n)$. Then for any $l \geq$ $u(n)$, the value of $f(n)$ can be computed from $n$ and $A \uparrow l$; hence

$$
n \leq C(f(n)) \leq^{+} C(A \uparrow l)+2 \log n,
$$

and thus for almost all $n$ and all $l \geq u(n)$, we have $n / 2 \leq C(A \uparrow l)$. As a consequence, a finite variation of the $A$-recursive order

$$
g: n \mapsto \max \{l: u(l) \leq n\} / 2
$$

witnesses that $A$ is autocomplex.

The next result shows that the notions of complex and autocomplex sets can be characterized in terms of the degrees of DNR functions. In the case of complex sets, one can recast this notion in terms of the degrees of recursively bounded DNR functions, because a complex set can tt-compute only functions which are recursively bounded. There are Turing degrees which contain DNR functions but no recursively bounded functions [1]; hence some Turing degrees consist of autocomplex sets without containing any complex set.

Theorem 2.3. Let $A$ be any set.

1. A is autocomplex iff $A$ computes a DNR function.

2. A is complex iff $A$ tt-computes a DNR function iff $A$ wtt-computes a DNR function.

Proof. 1. Suppose $A$ is not autocomplex, but computes a DNR function $\varphi_{r}^{A}$. There is a recursive function $e(\cdot)$ such that for any $x$ and $y$, if the universal machine $U$ on $x$ converges, and additionally $\varphi_{r}^{U(x)}(y)$ converges, then $\varphi_{e(x)}(y)=\varphi_{r}^{U(x)}(y)$. For each $n$, let $g(n)$ be the maximum of the use of all computations $\varphi_{r}^{A}(e(x))$ with $|x| \leq n$. Clearly $g$ is recursive in $A$. Since $A$ is not autocomplex, there must be some $n$ such that $C\left(A\lceil g(n)) \leq n\right.$. If $x_{n}$ is a witnessing code, i.e. $U\left(x_{n}\right)=A\left\lceil g(n),\left|x_{n}\right| \leq n\right.$, then $\varphi_{r}^{A}\left(e\left(x_{n}\right)\right)=\varphi_{e\left(x_{n}\right)}\left(e\left(x_{n}\right)\right)$. Thus $\varphi_{r}^{A}$ is not DNR.

Now suppose $A$ is autocomplex. Let $g$ be an $A$-order function such that for all $n, C(A\lceil n) \geq g(n)$. Let $\gamma$ be an $A$-recursive function such that $g(\gamma(n)) \geq n$ for all $n$. Let $f(n)=A\lceil\gamma(n) ; f$ is recursive in $A$. If $f(n)$ is infinitely often equal to $\varphi_{n}(n)$, then for such an $n, C(A \uparrow \gamma(n)) \leq \log n+c$ for some constant $c$. Letting $m=\gamma(n)$,

$$
C(A\lceil m) \leq \log n+c \leq \log g(m)+c<g(m)
$$

for sufficiently large $m$, which is a contradiction. Thus, we conclude that $f(n) \neq$ $\varphi_{n}(n)$ for all but finitely many $n$, so $f$ computes a DNR function.

2. This is obtained by the same proof; if $A w t t$-computes $\varphi_{r}^{A}$, then the function $g$ is recursive, and if $g$ is recursive, then $f$ is $t t$-computable from $A$.

Theorem 2.6 below gives a variety of useful characterizations of the complex sets. These characterizations are mainly based on the following definition and result of Jockusch.

Definition 2.4 (Jockusch [5]). A function $h: \omega \rightarrow \omega$ is called strongly DNR $(S D N R)$ if for all $x, h(x) \neq \varphi_{y}(y)$ for all $y \leq x$.

Theorem 2.5 (Jockusch [5]). Every DNR function computes an SDNR function.

The characterizations of complex sets and Jockusch's result can be extended to the following comprehensive characterization of complex sets. 
Theorem 2.6. For a set $A$ the following conditions are equivalent.

(1) There is an SDNR function $f_{1} \leq_{w t t} A$.

(2) For every recursive function $g$ there is a function $f_{2} \leq_{w t t} A$ such that for all recursive $h$ with $\forall n\left[\left|W_{h(n)}\right|<g(n)\right]$ it holds that $\forall^{\infty} n\left[f_{2}(n) \notin W_{h(n)}\right]$.

(3) There is $f_{3} \leq_{w t t} A$ such that for all partial-recursive $V$ with domain $\{0,1\}^{*}$ and almost all $n, f_{3}(n) \notin\left\{V(p): p \in\{0,1\}^{*} \wedge|p|<n\right\}$.

(4) There is $f_{4} \leq_{w t t} A$ with $C\left(f_{4}(n)\right) \geq n$ for all $n$.

(5) There is a DNR function $f_{5} \leq_{w t t} A$.

(6) $A$ is complex.

One can also write $f_{k} \leq_{t t} A$ in place of $f_{k} \leq_{w t t} A$ in these conditions.

Proof. (1) implies (2): Let a recursive function $g$ be given; without loss of generality $g$ is increasing. Furthermore there is a recursive function $a$ such that $\varphi_{a(e, n, m)}(x)$ is the $m$-th element enumerated into $W_{\varphi_{e}(n)}$ whenever $\left|W_{\varphi_{e}(n)}\right| \geq m$. There is a recursive function $b$ such that $b(k)>a(e, n, m)$ for all $e, m, n \leq g(k)$. Now let $f_{2}(n)=f_{1}(b(n))$. It follows from the definition of SDNR functions that for every total function $h=\varphi_{e}$ and all $n>e, f_{1}(b(n))$ differs from the first $g(n)$ elements of $W_{h(n)}$; in the case that $\left|W_{h(n)}\right|<g(n)$ it follows that $f_{2}(n) \notin W_{h(n)}$. Furthermore, as $f_{1} \leq_{w t t} A$ and $f_{2}$ is many-one reducible to $f_{1}, f_{2} \leq_{w t t} A$ as well. Hence $f_{2}$ satisfies the requirements asked for.

(2) implies (3): Here one can choose $g$ such that $g(n)=2^{n}$ and consider the corresponding $f_{2}$. Then one takes $f_{3}=f_{2}$ and

$$
W_{h(n)}=\left\{V(p): p \in\{0,1\}^{*} \wedge|p|<n\right\} .
$$

By assumption $f_{2}(n) \notin W_{h(n)}$ for almost all $n$; the same then also holds for $f_{3}$.

(3) implies (4): Let $f_{3}$ be given as in (3) and let $U$ be the universal machine on which $C$ is based. Then there is a constant $c$ such that for all $n \geq c$ and all $p$ in the domain of $U$ with $|p|<n, f_{3}(n) \neq U(p)$. Thus $C\left(f_{3}(n)\right) \geq n$ for all $n \geq c$. Now let $f_{4}(n)=f_{3}(n+c)$ in order to meet condition (4).

(4) implies (5): There is a constant $c$ with $C\left(\varphi_{n}(n)\right)<n+c$ for all $n$ such that $\varphi_{n}(n)$ converges. Taking $f_{4}$ as in $(4)$, the function $f_{5}$ given as $f_{5}(n)=f_{4}(n+c)$ satisfies that $C\left(\varphi_{n}(n)\right)<n+c \leq C\left(f_{5}(n)\right)$ for all $n$ where $\varphi_{n}(n)$ is defined. Hence (5) is satisfied.

(5) implies (6): This follows from Theorem 2.3

(6) implies (1): This is similar to the proof in Theorem 2.3. Just choose a constant $c$ again such that $C\left(\varphi_{n}(n)\right) \leq n+c$ whenever defined. It follows from the definition of a complex set that there is a recursive function $f_{6}$ with $C\left(A\left\lceil f_{6}(n)\right)>\right.$ $n+c$ for all $n$ and hence $f_{1}(n)=\left(A \uparrow f_{6}(n)\right)$ is an SDNR function.

This completes the equivalences. As all steps from $(k)$ to $(k+1)$ for $k=1,2,3,4$ make a many-one reduction from one function to another and as $f_{1} \leq_{t t} A$, these functions are all tt-reducible to $A$ along the lines of the proof. Hence all conditions hold with $f_{k} \leq_{t t} A$ in place of $f_{k} \leq_{w t t} A$.

One can easily see that the above characterizations hold with Turing reducibility in place of weak truth-table reducibility for autocomplex sets. Hence one obtains the following theorem as well.

Theorem 2.7. For a set $A$ the following conditions are equivalent.

(1) There is an SDNR function $f_{1} \leq_{T} A$. 
(2) For every recursive function $g$ there is a function $f_{2} \leq_{T} A$ such that for all recursive $h$ with $\forall n\left[\left|W_{h(n)}\right|<g(n)\right]$ it holds that $\forall^{\infty} n\left[f_{2}(n) \notin W_{h(n)}\right]$.

(3) There is $f_{3} \leq_{T} A$ such that for all partial-recursive $V$ with domain $\{0,1\}^{*}$ and almost all $n, f_{3}(n) \notin\left\{V(p): p \in\{0,1\}^{*} \wedge|p|<n\right\}$.

(4) There is $f_{4} \leq_{T} A$ with $C\left(f_{4}(n)\right) \geq n$ for all $n$.

(5) There is a DNR function $f_{5} \leq_{T} A$.

(6) $A$ is autocomplex.

Remark 2.8. In the two preceding theorems, condition (2) says that one can compute for each order a function avoiding all r.e. traces of given cardinality almost everywhere; the DNR Turing degrees can also be characterized as those where there is for each given r.e. trace a function avoiding this trace everywhere. Condition (3) is the r.e. counterpart of Theorem [5.1 (5) below. Condition (4) says that one can compute a function which takes on all inputs a value of sufficiently high Kolmogorov complexity. Condition (6) is already known to be equivalent to the other ones, but it was convenient to construct the proof from (5) to (1) through (6) in Theorem 2.6.

Remark 2.9. Instead of computing a lower bound $h$ for the complexity of $A$ from $A$ as an oracle, one can also formulate the same result by making a function $h$ mapping strings to lower bounds; this function then only needs to be correct on strings stemming from the characteristic function of $A$. The characterization is the following:

$A$ is complex iff there is a recursive function $h$ such that

- for all $\sigma, \tau \in\{0,1\}^{*}, h(\sigma \tau) \geq h(\sigma)$ and

- for all $y, h(A\lceil y) \leq C(A\lceil y)$ and

- for every $n$, the set of strings $\sigma \in\{0,1\}^{*}$ with $h(\sigma) \leq n$ is finite.

Similarly $A$ is autocomplex iff there is a function $h \leq_{T} A$ with these same properties.

Remark 2.10. M.I. Kanovič [6,7] (see Li and Vitányi [10, Exercise 2.7.12, p. 184) states a result to the effect that the notions of being complex and autocomplex, defined in terms of monotonic complexity, are the same as being wtt-complete and T-complete in the special case of recursively enumerable sets.

\section{Hyperavoidable and effectively immune sets}

Miller 11 introduced the notion of a hyperavoidable set. A set $A$ is hyperavoidable iff it differs from all characteristic functions of recursive sets within a length computable from a program of that recursive set. For random sets, this length is at most the length of the program plus a constant. So hyperavoidable sets are a generalization of random sets, and Theorem 3.3 shows that one can characterize that $A$ is hyperavoidable similarly to the way one characterizes that $A$ is random in terms of prefix-free Kolmogorov complexity: $A$ is hyperavoidable iff there is an order function $g$ such that $\forall x(C(A\lceil x) \geq g(x))$.

Definition 3.1. A set of nonnegative integers $A$ is called hyperavoidable if there is an order function $h$ such that for all $x$ with

$$
\{0,1, \ldots, h(x)-1\} \subseteq\left\{y: \varphi_{x}(y) \downarrow \in\{0,1\}\right\},
$$

we have $A \uparrow h(x) \neq \varphi_{x} \uparrow h(x)$. In other words,

$$
\forall x \exists y<h(x) A(y) \neq \varphi_{x}(y) .
$$


Note that if $A$ is hyperavoidable via $h$ and $\tilde{h}$ is a further order function with $\tilde{h}(x) \geq h(x)$ for all $x$, then $A$ is also hyperavoidable via $\tilde{h}$.

The original reason for the interest in hyperavoidability is the following result.

Theorem 3.2 ([11, Theorem 4.6.4]). A set is hyperavoidable iff it is not wttreducible to any hyperimmune set.

Theorem 3.3. For a set $A$ the following statements are equivalent:

(1) A is complex.

(2) A is hyperavoidable.

Proof. (1) implies (2): If $A$ is complex, then as we have seen, $\varphi_{r}^{A}$ is a DNR function for a wtt-reduction $\varphi_{r}$. Assume that $u(x)$ is the use of the wtt-reduction at input $x$, that is, the maximal element queried at the computation of $\varphi_{r}^{A}(x)$; note that this element is independent of the oracle $A$. Furthermore, for any $n$, let $W_{n}=$ $\left\{z: \varphi_{n}(z) \downarrow=1\right\}$. Now let $\varphi_{s(n)}(x)=\varphi_{r}^{W_{n}}(x)$ iff $\varphi_{n}(z) \downarrow \in\{0,1\}$ for all $z \leq$ $u(x)$ and the computation $\varphi_{r}^{W_{n}}(x)$ terminates; let $\varphi_{s(n)}(x)$ be undefined otherwise. Define $h(n)=u(s(n))+1$. Since $\varphi_{r}^{A}$ is a DNR function there is no $n$ such that $\varphi_{s(n)}(s(n)) \downarrow=\varphi_{r}^{A}(s(n))$. Thus for every $n$ there is a $y \leq u(s(n))$ such that $\varphi_{n}(y) \uparrow$ or $\varphi_{n}(y) \neq A(y)$. It follows that for every $n$ the function $\varphi_{n}$ differs from the characteristic function of $A$ before $h(n)$.

(2) implies (1): Suppose $A$ is not complex. Let $U$ be the universal function on which $C$ is based. Let $f$ be a total recursive function such that, for all $\sigma \in\{0,1\}^{*}$ where $U(\sigma)$ is defined, $\varphi_{f(\sigma)}$ is the characteristic function of the set $\{x: U(\sigma)(x) \downarrow$ $=1\}$. Furthermore, let $\tilde{f}(n)=\max \left\{f(\sigma): \sigma \in\{0,1\}^{*} \wedge|\sigma| \leq n\right\}$.

Let $h$ be any order function. Let $\tilde{h}(n)$ be the maximal $m$ with $m=0 \vee h(\tilde{f}(m)) \leq$ $n$. Then $\tilde{h}$ is also an order function. Thus there is a $y$ such that $C(A\lceil y)<\tilde{h}(y)$. Then there is a program $\sigma$ for the universal machine $U$ with $U(\sigma)=A \uparrow y$ and $|\sigma|<\tilde{h}(y)$. It follows that $h(f(\sigma))<y$. Thus the characteristic functions of $A$ and $\{x: U(\sigma)(x) \downarrow=1\}$ both coincide with $\varphi_{f(\sigma)}$ on the first $y$ inputs and so $A$ is not hyperavoidable via $h$. Since the choice of $h$ was arbitrary, $A$ is not hyperavoidable.

Miller [1] investigated the relation between hyperavoidable sets and effectively immune sets. A set $A$ is immune if it has no infinite recursive subset and effectively immune if there is a partial recursive function $\psi$ such that for all $e$, if $W_{e} \subseteq A$, then $e$ is in the domain of $\psi$ and $\left|W_{e}\right| \leq \psi(e)$. If there is no recursive function $f$ with $\forall n(|A \cap\{0,1,2, \ldots, f(n)\}| \geq n)$, then $A$ is called hyperimmune.

Theorem 3.4 ([11, Theorem 4.5.3]). Any effectively immune, nonhyperimmune set is hyperavoidable.

The converse is false; if $A$ is a complex set, then $A \oplus \omega$ is still complex, hence hyperavoidable, but not immune, hence certainly not effectively immune. However, up to truth-table degree, we shall see that the converse does hold.

Theorem 3.5. If $A$ is hyperavoidable, then there is a set $B \equiv_{t t} A$ which is effectively immune but not hyperimmune. This set can be viewed as a set of strings and is given as $B=\{A(0) A(1) \ldots A(n): n \in \omega\}$.

Proof. Obviously $B \equiv_{t t} A$. Furthermore, $B$ is not hyperimmune as $B$ contains a binary string of every length. Furthermore, it is effectively immune: As $A$ is complex there is a recursive function $\psi$ such that the complexity of all initial segments 
of $A$ which are longer than $\psi(n)$ is above $2 n$. Hence, whenever $\left|W_{e}\right|>\psi(e)$ and $W_{e} \subseteq B$, one can find effectively in $e$ a string $\theta(e) \in W_{e}$ which has at least the length $\psi(e)$. Now $C(\theta(e)) \leq e+c$ for some constant $c$ and all $e$ in the domain of $\theta$. On the other hand $C(\theta(e)) \geq 2 e$ for all $e$ in the domain of $\theta$ with $W_{e} \subseteq B$; so it can only happen for $e \leq c$ that $W_{e} \subseteq B \wedge\left|W_{e}\right| \geq \psi(e)$. A finite modification of $\psi$ makes $\psi$ to be a witness for $B$ being effectively immune.

Note that the proof actually shows that $B$ is strongly effectively immune. Hence one gets the following corollary.

Corollary 3.6. A set is complex iff its truth-table degree contains a set which is strongly effectively immune but not hyperimmune.

Remark 3.7. It is well known that a Turing degree contains a DNR function iff it contains an effectively immune set. In other words, a set is autocomplex iff its Turing degree contains an effectively immune set.

\section{Completions of Peano arithmetic}

Theorem 2.6 (4) shows that DNR is equivalent to the ability to compute a function $F$ such that $C(F(n)) \geq n$ for all $n$. The next result shows that if one enforces the additional constraint $F(n) \in\{0,1\}^{n}$, then one obtains the smaller class of PA-complete degrees instead of the DNR ones. Recall that $A$ has PA-complete degree iff $A$ computes a DNR function with a finite range. As Jockusch [5] showed, one can specify this range to be any given finite set as long as this set has at least 2 elements.

Theorem 4.1. The following is equivalent for every set $A$.

(1) A computes a lower bound $B$ of the plain complexity $C$ such that for all $n$ there are at most $2^{n}-1$ many $x$ with $B(x)<n$.

(2) A computes a function $F$ such that for all $n, F(n)$ has length $n$ and satisfies $C(F(n)) \geq n$

(3) A computes a DNR function D which has a fixed finite set as range.

Proof. (1) implies (2): $F(n)$ is just the lexicographically first string $y$ of length $n$ such that $B(y) \geq n$. This string exists by the condition that there are at most $2^{n}-1$ strings $x$ with $B(x)<n$. Since $B$ is a lower bound for $C$, one has that $C(F(n)) \geq n$ for all $n$. Furthermore, $F$ is computed from $B$.

(2) implies (3): There is a partial recursive function $\psi$ such that $\psi(x)=x \varphi_{n}(n)$ if $n$ is the length of $x$ and $\varphi_{n}(n)$ is defined. Furthermore there is a constant $c$ such that $C(\psi(x))<n+c$ for all $x, n$ with $x \in\{0,1\}^{n}$. Now one defines that $D(n)$ consists of the last $c$ bits of $F(n+c)$; this function is computed from $F$. Let $x$ be the first $n$ bits of $F(n+c)$ and assume that $\varphi_{n}(n)$ is defined. Then $C(\psi(x))<n+c$ and $x D(n)=F(n+c) \neq \psi(x)=x \varphi_{n}(n)$. Thus $D$ is a DNR function and its range is the finite set $\{0,1\}^{c}$.

(3) implies (1): Since $D \leq_{T} A$ is a DNR function with a finite range, the set $A$ is PA-complete by a result of Jockusch [5]. Thus there is a set $G \leq_{T} A$ which extends the graph of the universal function $U$ on which $C$ is based. $G$ satisfies the following two $\Pi_{1}^{0}$ conditions:

- $\forall p, x, s(U(p) \downarrow=x$ at stage $s \Rightarrow(p, x) \in G)$;

- $\forall p, x, y((p, x) \in G \wedge(p, y) \in G \Rightarrow x=y)$. 
Now one defines $B(x)=\min \{|p|:(p, x) \in G\}$. By standard Kolmogorov complexity arguments, it follows that $B$ is a lower bound for $C$ and that there are at most $2^{n}-1$ many $x$ with $B(x)<n$ for all $n$.

One might ask whether one can strengthen condition (2) in Theorem 4.1 and actually compute a string of maximal plain Kolmogorov complexity for every length $n$ from any PA-complete oracle. The answer to this question is that it depends on the universal machine on which the plain complexity is based. That is, for every r.e. oracle $B$ one can compute a corresponding universal machine which makes this problem hard not only for PA but also for $B$.

Theorem 4.2. For every recursively enumerable oracle $B$ there is a universal machine $U_{B}$ such that the following two conditions are equivalent for every oracle A:

- A has PA-complete degree and $A \geq_{T} B$.

- There is a function $F \leq_{T} A$ such that for all $n$ and for all $x \in\{0,1\}^{n}$, $F(n) \in\{0,1\}^{n}$ and $C_{B}(F(n)) \geq C_{B}(x)$, where $C_{B}$ is the plain Kolmogorov complexity based on the universal machine $U_{B}$.

Proof. Given any universal machine $U$ and r.e. set $B$, the value $U_{B}(p)$ takes the first case where there is a $q$ satisfying the corresponding condition:

- If $p=0 q$ and $U(q)$ is defined and has output $x$ and $|x|>|p|+2$, then $U_{B}(p)=x$.

- If $p=10 q$ and $q \in\{0,1\}^{*} \cdot\{0\}$ and $|q| \in B$, then $U_{B}(p)=q$.

- If $p=110 q$ and $q \in\{0,1\}^{*} \cdot\{1\}$, then $U_{B}(p)=q$.

- If $p=1110 q$, then $U_{B}(p)=q$.

- In all other cases, $U_{B}(p)$ is undefined.

First it is verified that $U_{B}$ is a universal machine. If $C(x) \geq|x|-3$, then $C_{B}(x) \leq$ $C(x)+7$ since $U_{B}(1110 x)=x$ and $C_{B}(x) \leq|x|+4$ for all $x$. If $C(x)<|x|-3$, then there is a program $q$ with $U(q)=x \wedge|q|<|x|-3$. Taking $p=0 q$, one has $|x|>|p|+2$ and $U_{B}(p)=x$. So $C_{B}(x) \leq C(x)+1 \leq C(x)+7$ again. Thus $U_{B}$ is a universal machine and $C_{B}$ a legitimate choice for the plain Kolmogorov complexity.

Note that for $U_{B}$, there are at most $2^{n-1}-1$ many strings $x$ of length $n$ with $C_{B}(x)<n-1$. Assume that $n>0, x \in\{0,1\}^{n}$ and $C_{B}(x) \geq n-1$. There are three cases:

- If $x \in\{0,1\}^{n-1} \cdot\{1\}$, then $C_{B}(x)=n+3$.

- If $x \in\{0,1\}^{n-1} \cdot\{0\}$ and $n \notin B$, then $C_{B}(x)=n+4$.

- If $x \in\{0,1\}^{n-1} \cdot\{0\}$ and $n \in B$, then $C_{B}(x)=n+2$.

Note that for each length $n$ there are strings ending with 0 and ending with 1 which satisfy $C_{B}(x) \geq|x|-1$. So given $n$, let $x \in\{0,1\}^{n}$ have maximal plain complexity $C_{B}$.

- If $n \in B$, then $x \in\{0,1\}^{n-1} \cdot\{1\}$ and $C_{B}(x)=n+3$.

- If $n \notin B$, then $x \in\{0,1\}^{n-1} \cdot\{0\}$ and $C_{B}(x)=n+4$.

On one hand, if $F \leq_{T} A$ and $F$ is as defined in the statement of this theorem and $n>0$, then the last bit of $F(n)$ is equal to $B(n)$. Thus $B \leq_{T} A$. Furthermore, $A$ is PA-complete since $C_{B}(F(n)) \geq n$ for all $n$.

On the other hand, if $B \leq_{T} A$, then one can compute the maximal plain complexity of a string of length $n$ which is $n+4-B(n)$. Having this number, one can use the PA-completeness of $A$ to find a string $x$ of length $n$ such that $C_{B}(x) \geq n+4-B(n)$. This gives the desired equivalence. 
While PA-completeness can be characterized in terms of $C$, the obvious analogues fail for $H$. First one cannot replace $C$-incompressible by $H$-incompressible since one can compute relative to any random set $A$ the function mapping $n$ to the $H$-incompressible string $A(0) \ldots A(n)$. So one would like to know whether the oracles $A$ which permit us to compute strings of maximal complexity would characterize the PA-complete degrees. But, instead, the corresponding notion gives a characterization of the halting problem $K$. Note that the following theorem is independent of the underlying universal machine.

Theorem 4.3. For any set $A, A \geq_{T} K$ iff there is a function $F \leq_{T} A$ such that $\forall n \forall x \in\{0,1\}^{n}\left(F(n) \in\{0,1\}^{n} \wedge H(x) \leq H(F(n))\right)$.

Proof. Since $H$ is $K$-recursive, such an $F$ can obviously be computed if $A \geq_{T} K$. For the remaining direction, assume that $F$ is as in the statement of the theorem and $F \leq_{T} A$. The proof consists now of three parts:

- First, a sequence of partitions is constructed to be used later.

- Second, it is shown that there is a constant $k$ such that for every $n$ and every $m>n+k+1$ the binary number $b v(y)$ consisting of the last $2 k$ bits $y$ of the string $F\left(2^{m}+n\right)$ satisfies that $P_{m, b v(y)}$ does not contain $H(n)$.

- Third, it is shown how the fact from the second statement can be used to prove that $K \leq_{T} A$.

First, let $P_{0}, P_{1}, P_{2}, \ldots$ be an enumeration of all primitive recursive permutations of the integers and let $P_{m, o}$ be the $o$-th member of the permutation; here $P_{m, o}=\emptyset$ in the case that the permutation has less than $o$ nonempty members. Note that every partition has infinitely many indices.

Second, let $U$ be the universal machine on which $H$ is based and let $\tilde{U}(p)=n$ whenever $U(p)=2^{m}+n$ for some $m>n$. It is known [9, Section 4.3] that there is a constant $c_{1}$ such that

$$
2^{-H(n)} \geq \sum_{p \text { with } \tilde{U}(p)=n} 2^{-|p|-c_{1}}
$$

for all $n$. Now, let $n$ be given and $p$ be of length $H(n)$ such that $U(p)=n$; that is, let $p$ be a minimal program for $n$. As the sum of $2^{-|q|-c_{1}}$ over $q$ with $\exists m>n\left(U(q)=2^{m}+n\right)$ is bounded by 1 , there is, uniformly in $p$, a prefix-free machine $V_{p}$ which is conditionally universal in the following sense: For all $m>n$ there is a $q$ with $|q|=H\left(2^{m}+n\right)+c_{1}-|p| \wedge V_{p}(q)=2^{m}+n$. If $p$ is not a minimal program for any $n$, then nothing is required except that $V_{p}$ is prefix-free. This permits us to construct the following machine $V: V(r)=V_{p}(q)$ iff $r=p q, U(p)$ is defined and $V_{p}(q)$ is defined; if $r$ cannot be split in $p, q$ this way, then $V(r)$ is undefined; note that the splitting of $r$ into $p, q$ is unique whenever it is possible. The main properties of $V$ are the following:

- $V$ is prefix-free;

- for all $n$ and all $m>n$ there are $p, q$ such that $V(p q)=2^{m}+n, H(n)=|p|$, $U(p)=n$ and $|p q| \leq H\left(2^{m}+n\right)+c_{1}$.

Based on $V$ one constructs a further prefix-free machine $W$ such that $W(r)=z$ iff there are $x, y, m, n, p, q, k$ such that the following conditions are satisfied:

- $z=x y$;

- $m>n+k+1$;

- $|x y|=2^{m}+n$ and $|y|=2 k$; 
- $r=p q 1^{k} 0 x$

- $U(p)$ is defined and takes the value $n$;

- $V_{p}(q)$ is defined and takes the value $2^{m}+n$;

- the binary value $b v(y)$ satisfies that $|p| \in P_{m, b v(y)}$.

Here $b v(y)$ is the binary value of $y$, for example, $b v(000101)=5$. Note that $x, y, m, n, p, q, k$ depend uniquely on $r$ whenever $W(r)$ can be defined by an appropriate choice of the parameters.

One can see from the definition that $W$ is prefix-free. Hence there is a constant $c_{2}$ such that $H(W(r)) \leq|r|+c_{2}$ for all $r$ in the domain of $W$.

Furthermore, note that $H\left(F\left(2^{m}+n\right)\right) \geq H\left(2^{m}+n\right)+2^{m}+n-c_{3}$ for some constant $c_{3}$. To see this, note that the sum of $2^{-H(u)}$ over all $u \in\{0,1\}^{\ell}$ is at most $2^{c_{4}-H(\ell)}$ for some constant $c_{4}$ independent of $\ell$. As there are $2^{\ell}$ such $u$, it holds that $H(u) \geq H(\ell)+\ell-c_{4}$ for at least one of these $u$. Now one can take $c_{3}=c_{4}$ and use that $F$ takes a string of maximal prefix-free Kolmogorov complexity to get the desired statement.

Fix the value of the parameter $k$ from now onward as

$$
k=c_{1}+c_{2}+c_{3}+2
$$

and note that for all $r$ in the domain of $W$ having this fixed parameter $k$ it holds that $H(W(r))<H(F(|W(r)|))$.

Now the second part is completed by showing that whenever $F\left(2^{m}+n\right)=x y$ with $m>n+k+1 \wedge|y|=2 k$, then $H(n) \notin P_{m, b v(y)}$. So assume by way of a contradiction that $m>n+k+1, F\left(2^{m}+n\right)=x y,|y|=2 k$ and $H(n) \in P_{m, b v(y)}$.

Let $p$ be a program with $U(p)=n \wedge|p|=H(n)$. Let $q$ be such that $2^{m}+n=$ $V(p q)$ and $|q| \leq H\left(2^{m}+n\right)+c_{1}-|p|$; the existence of such a $q$ had been shown above when constructing the machine $V_{p}$. One can verify that for the input $r=p q 1^{k} 0 x$ and $z=x y$ the computation $W(r)$ converges to $z$ as in the definition of $W(r)$, the first three search conditions on $r, z$ are satisfied by the choice of the above parameters, $p, q$ are selected such that the fourth and fifth search conditions are satisfied and the assumption $H(n) \in P_{m, b v(y)}$ gives that the sixth search condition is satisfied.

Now a contradiction is derived by showing that the two conditions on $H(x y)$ are not compatible. On one hand, $r=p q 1^{k} 0 x$ and

$$
\begin{aligned}
|r| & =|p q|+\left|1^{k}\right|+|0|+|x| \leq\left(H\left(2^{m}+n\right)+c_{1}\right)+k+1+|x| \\
& =\left(H\left(2^{m}+n\right)+c_{1}\right)+k+1+\left(2^{m}+n-2 k\right) \\
& =H\left(2^{m}+n\right)+2^{m}+n+1+c_{1}-k \\
& \leq H\left(2^{m}+n\right)+2^{m}+n-c_{2}-c_{3}-1 ;
\end{aligned}
$$

hence $H(x y)=H(W(r)) \leq|r|+c_{2} \leq H\left(2^{m}+n\right)+2^{m}+n-c_{3}-1$. On the other hand, $H(x y)=H\left(F\left(2^{m}+n\right)\right) \geq H\left(2^{m}+n\right)+2^{m}+n-c_{3}$. This contradiction establishes that it does not happen that $H(n) \in P_{m, b v(y)}$ for any $n$ and $m>n+k+1$ with $y$ being the last $2 k$ bits of $F\left(2^{m}+n\right)$.

Third, one can run the following $A$-recursive algorithm to determine for any given $n$ a set of up to $4^{k}-1$ elements which contains $H(n)$ by the following algorithm. Here $c_{5}$ is a constant such that $H(n) \leq n+c_{5}$ for all $n$.

- Let $E=\left\{0,1, \ldots, n+c_{5}\right\}$ and $m=n+k+2$. 
- While $|E| \geq 4^{k}$ Do Begin $m=m+1$, Determine the string $y$ consisting of the last $2 k$ bits of $F\left(2^{m}+n\right)$

and update $E=E-P_{m, b v(y)}$ End.

- Output E.

This algorithm terminates since whenever $|E| \geq 4^{k}$ at some stage $m$, then there is $o>m$ such that the first $4^{k}$ members of $P_{o}$ all intersect $E$ and one of them will be removed so that $E$ loses an element in one of the stages $m+1, \ldots, o$. Thus the above algorithm computes relative to $A$ for input $n$ a set of up to $4^{k}-1$ elements containing $H(n)$. By a result of Beigel, Buhrman, Fejer, Fortnow, Grabowski, Longpré, Muchnik, Stephan and Torenvliet [2, such an $A$-recursive algorithm can only exist if $K \leq_{T} A$.

Remark 4.4. Calude [3] had circulated the following question: If $A$ is an infinite set of strings of maximal $H$-complexity, that is, if $A$ satisfies

$$
\forall x \in A \forall y[|y|=|x| \Rightarrow H(y) \leq H(x)],
$$

is then $K \leq_{T} A$ ? The question remains open until today, but the above theorem gives a partial answer to this question as it shows that $K \leq_{T} A$ is true for all sets $A$ of maximal $H$-complexity which contain at least one string of each length.

Nies [12] pointed out to the authors that one might study the analogue of incompressible strings in the sense that one looks at functions $F$ producing strings of length $n$ and approximate complexity $n+H(n)$. More precisely, the proof of the above theorem also shows the more general result that for any oracle $A, A \geq_{T} K$ if and only if there is a function $F \leq_{T} A$ and a constant $c$ such that for all $n$, $F(n) \in\{0,1\}^{n}$ and $H(F(n)) \geq n+H(n)-c$.

A related question to the one of Calude is whether there is an infinite set $B$ such that $K \not_{T}\{\langle n, H(n)\rangle: n \in B\}$. If such a set exists, one can consider a constant $c$ such that there is a set $A$ which contains for each $b \in B$ exactly one $a \in\{0,1\}^{b}$ with $H(a) \geq H(b)+b-c$. Given $B$ and $c$, such a set $A$ can be constructed relative to any oracle which is PA-complete relative to $B$; note that such oracles need not be above $K$. Hence Nies' version of the question of Calude has a negative answer in the case that this set $B$ exists.

\section{Characterizing high or DNR Degrees}

In this section various characterizations are obtained for when a Turing degree is high or DNR, in terms of what are called eventually different functions. The study of such functions originates in set theory where set theorists had defined that a function $f: \omega \rightarrow \omega$ is eventually different iff for each $g: \omega \rightarrow \omega$ in the ground model, $\{x: f(x)=g(x)\}$ is finite. A computability-theoretic analogue is obtained by replacing the ground model by the set of recursive functions. The corresponding Turing degrees form the union of the high and the DNR degrees and also admit a characterization in terms of upper bounds on Kolmogorov complexity. Note that this analogue is a relaxed version of SDNR functions as every SDNR function is eventually different from every partial-recursive function and every function eventually different from all partial-recursive ones is DNR.

Theorem 5.1 has been applied by Stephan and Yu [13, and Greenberg and Miller [4. 
Theorem 5.1. The following statements are equivalent:

(1) A computes a function $f$ that is eventually always different from each recursive function.

(2) A computes a function $g$ such that either $g$ dominates each recursive function or $g$ is eventually always different from each partial recursive function.

(3) $A$ is of high or DNR Turing degree.

(4) A computes an unbounded function $f$ which is dominated by all recursive upper bounds on $C$.

(5) A computes a function $F$ such that for every total recursive $V$ with domain $\{0,1\}^{*}$ and almost all $n, F(n) \notin\left\{V(p): p \in\{0,1\}^{*} \wedge|p|<n\right\}$.

Proof. (1) implies (2): If $A$ has high degree, then $A$ computes a function $g$ dominating all recursive functions (we can either take this as our definition of high degree, or invoke Martin's Theorem from 1966) and (2) is satisfied. So assume that $A$ does not have high degree and let $f \leq_{T} A$ be eventually different from all total recursive functions. For a contradiction suppose $\varphi_{d}$ is some partial recursive function and $f(x)=\varphi_{d}(x)$ on infinitely many inputs $x$ in the domain of $\varphi_{d}$. Let $p$ be a function such that for each $n$, there are $n+1$ many $x$ for which $f(x)=\varphi_{d, p(n)}(x)$. (Here $\varphi_{e, s}(x)$ is the value of $\varphi_{e}(x)$ after $s$ steps.)

Clearly $p \leq_{T} f$. Hence as $f$ is not of high degree, there is a recursive nondecreasing function $q$ such that for infinitely many $n, q(n) \geq p(n)$. Now define a total recursive function $\varphi_{e}$ by $\varphi_{e}(n)=\varphi_{d, q(n)}(n)$ if this computation halts, and $\varphi_{e}(n)=0$ otherwise. Now suppose $q(n) \geq p(n)$. Then for some $k \geq n, \varphi_{d, q(n)}(k)=f(k)$. So $\varphi_{d, q(k)}(k)=f(k)$ and hence $\varphi_{e}(k)=f(k)$. Since there are infinitely many such $n$, there are infinitely many such $k$, and hence $f$ agrees with a total recursive function on infinitely many inputs.

(2) implies (3): Let $g \leq_{T} A$ have the desired properties. If $f$ dominates every recursive function, then $A$ has high Turing degree. If $g$ is eventually different from all partial recursive functions, then consider any $e$ such that $\varphi_{e}(x)=\varphi_{x}(x)$ for each $x$. Then a finite modification of $f$ is DNR; hence $A$ has DNR degree.

(3) implies (4): First assume the case that $A$ is of high Turing degree. Then there is a function $g \leq_{T} A$ which grows faster than every recursive function. Taking $U$ to be the universal machine, then $f(x)$ is the length of the shortest program $p$ such that $U(p)=x$ within $g(x)$ steps. If $\tilde{C}$ is a recursive upper bound of $C$, then let $t(x)$ be the time to compute $U(p)$ for the fastest program $p$ of length up to $\tilde{C}(x)$ with $U(p)=x$. The function $t$ is recursive and dominated by $g$; thus $f(x) \leq \tilde{C}(x)$ for almost all $x$. So $\tilde{C}$ dominates $f$.

Second assume the case that $A$ is of DNR Turing degree. Then by Theorem 2.6 (4) there is a function $g \leq_{T} A$ such that $C(g(n)) \geq n$ for all $n$. Without loss of generality, $f(n)$ is a string of length at least $n$ for every $n$. Now define for every $x \in\{0,1\}$ the value $f(x)$ as the maximum of all $m \leq n$ such that either $m=0$ or $x=g(m)$. Clearly $f$ is unbounded as $f(g(m)) \geq m$ for all $m$. Furthermore, $f$ is dominated by $C$ and hence also by all upper bounds $\tilde{C}$ of $C$.

(4) implies (5): Let $f$ be the given lower bound. Now define $F(n)$ to be the length-lexicographically first string $x$ with $f(x) \geq 2 n$ which exists by the assumption that $f$ is unbounded.

Let $V$ be recursive with domain $\{0,1\}^{*}$. There is a constant $c$ such that $C(V(p))$ $\leq|p|+c$ for all programs $p$ and $C(x) \leq|x|+c$ for all $x$. Now let $\tilde{C}$ be the minimum 
of $|p|+c$ for all programs $p$ with either $|p|=|x|$ or $V(p)=x$. $\tilde{C}$ is a recursive upper bound for $C$. Thus, for sufficiently large $n$ and all $p$ of length up to $n$, $\tilde{C}(F(n)) \geq 2 n>n+c$ and so $V(p) \neq F(n)$.

(5) implies (1): Given any function $h$, define for all strings $p$ of length $n-1$ that $V(p)=h(n)$. It follows that the function $F$ differs from $h$ almost always and so (1) is satisfied.

A similar result can be obtained for the Turing degrees of Peano complete or high sets.

Theorem 5.2. The following statements are equivalent for any set $A$ :

(1) A has either high or PA-complete Turing degree.

(2) A computes a function $B$ such that $\forall n\left(\left|\left\{x \in\{0,1\}^{*}: B(x)=n\right\}\right| \leq 2^{n}\right)$ and $B$ is dominated by all recursive upper bounds of $C$.

(3) A computes a function $F$ mapping every $n$ to a string of length $n$ such that for every recursive upper bound $\tilde{C}$ on $C$ and for almost all $n, \tilde{C}(F(n)) \geq n$.

(4) A computes a $\{0,1\}$-valued function $g$ such that for all infinite recursive subsets $R$ of the domain of the diagonal function, $\forall^{\infty} n \in R\left(g(n) \neq \varphi_{n}(n)\right)$.

Proof. (1) implies (2): If $A$ has PA-complete degree, then $B$ exists by Theorem 4.1. If $A$ has high degree, then let $d \leq_{T} A$ be a function which dominates all recursive functions and let $B(x)=C_{d(|x|)}(x) . B$ is an upper bound of $C$ and thus satisfies the cardinality condition. Furthermore, if $\tilde{C}$ is a recursive upper bound on $C$, then the function mapping $n$ to the first $s$ such that $C_{s}(x) \leq \tilde{C}(x)$ for all $x \in\{0,1\}^{*}$ with $|x| \leq n$ is recursive and thus dominated by $d$. It follows that $B(x) \leq \tilde{C}(x)$ for almost all $x$ and so (2) is satisfied.

(2) implies (3): Take $B$ as specified for (2) and let $F(n)$ be the lexicographic first string $x$ of length $n$ with $B(x) \geq n$. This string exists since there are at most $2^{n}-1$ many strings $y$ with $B(y)<n$. Note that $F \leq_{T} A$ since $B \leq_{T} A$. Since every recursive upper bound $\tilde{C}$ dominates $B$, condition (3) is satisfied.

(3) implies (1): Assume that $A$ does not have PA-complete Turing degree. Then by Theorem 4.1, there are infinitely many $n$ with $C(F(n))<n$. Then there is an increasing $A$-recursive function $d$ such that for all $n$ there is $m \geq n$ with $C_{d(n)}(F(m))<m$. In particular, if $h$ is also increasing and $h(n) \geq d(n)$ infinitely often, then $C_{h(m)}(F(m))<m$ for infinitely many $m$. So the mapping from $x$ to $C_{h(|x|)}(x)$ cannot be recursive and $h$ cannot be a recursive function. Thus $d$ dominates every recursive function and $A$ has high Turing degree.

(1) implies (4): If $A$ has PA-complete degree, then it is well known that there is a $\{0,1\}$-valued DNR function $g \leq_{T} A$. If $A$ has high degree, then one can again take an $A$-recursive function $d$ dominating all recursive ones and consider the function $g$ with $g(n)=1 \Leftrightarrow \varphi_{n, d(n)}(n) \downarrow=0$. If $R$ is a recursive subset of the domain of the diagonal function, then $d$ dominates the time which $\varphi_{n}(n)$ needs to converge on inputs from $R$ and thus $g(n)=\varphi_{n}(n)$ only for finitely many $n$ in $R$.

(4) implies (1): This is similar to the implication from (3) to (1). Assume that $A$ does not have PA degree. Let $g \leq_{T} A$ be $\{0,1\}$-valued. There are infinitely many $n$ in the domain of the diagonal function with $g(n)=\varphi_{n}(n)$. There is an increasing $A$-recursive function $d$ such that for all $n$ there is $m \geq n$ with $g(m)=\varphi_{m, d(n)}(m)$. For a given increasing, recursive function $h$, let $R=\left\{n: \varphi_{n, h(n)}(n) \downarrow\right\}$. Whenever $h(n) \geq d(n)$, then there is $m \geq n$ with $\varphi_{m, d(n)}(m) \downarrow=g(m)$. Furthermore, $m \in R$ since $d(n) \leq h(n) \leq h(m)$. Since $g$ is correct on only finitely many elements of $R$, 
$h(n)<d(n)$ for almost all $n$ and $d$ dominates every recursive function. Thus $A$ has high Turing degree.

Theorem 5.3. If a set $A$ has high Turing degree, then there is a function $F \leq_{T} A$ mapping every $n$ to a string of length $n$ such that

$$
\forall x \in\{0,1\}^{n}(H(x) \leq \tilde{H}(F(n)))
$$

for every recursive upper bound $\tilde{H}$ of $H$ and almost all $n$.

Proof. Let $A$ have high degree. There is a function $f \leq_{T} A$ which dominates all recursive functions. Let $F(n)$ be the lexicographically first $x \in\{0,1\}^{n}$ for which $H_{f(n)}(x)$ is maximal. Now, let $\tilde{H}$ be a recursive upper bound on $H$. The function mapping $n$ to the first $s$ such that $H_{s}(x) \leq \tilde{H}(x)$ for all $x \in\{0,1\}^{n}$ is recursive and thus dominated by $f$. It follows that $H_{f(n)}(F(n)) \leq \tilde{H}(F(n))$ for almost all $n$. On the other hand, $H(x) \leq H_{f(n)}(F(n))$ for all $n$, so the statement of the theorem is satisfied in the case that $A$ has high Turing degree.

\section{R.E. TRACEABLE SETS}

It was shown in 8 that a set $A$ is r.e. traceable if and only if every Martin-Löf random set is Schnorr random relative to $A$. We remind the reader of the definitions. (Recall that $W_{n}$ is the $n$-th r.e. set, and $D_{n}$ the finite set with canonical index $n$.)

Definition 6.1. A set $A$ is r.e. traceable if there is a recursive function $p$ such that for all $f \leq_{T} A$, there is a recursive function $g$ such that for all $n, f(n) \in W_{g(n)}$ and $W_{g(n)}$ has at most $p(n)$ elements. Similarly, $A$ is recursively traceable if the same statement holds with the r.e. set $W_{g(n)}$ replaced by the canonically finite set $D_{g(n)}$.

We now characterize r.e. traceable sets as being "uniformly very far from DNR". A similar characterization holds for recursively traceable sets, and shows that recursively traceable sets compute no eventually different function.

Theorem 6.2. The following statements are equivalent for any set $A$ :

(1) A is r.e. traceable.

(2) There is a fixed recursive function $z(n)$ such that for each $f \leq_{T} A$, and almost every $n$, the set $\left\{x: f(x)=\varphi_{x}(x)\right\}$ has at least $n$ elements below $z(n)$.

The following statements are equivalent for any set $A$ :

(3) A is recursively traceable.

(4) There is a fixed recursive function $g$ such that for each $f \leq_{T}$ A, there is a recursive function $\varphi_{e}$ such that for almost every $n$, the set $\{x: f(x)=$ $\left.\varphi_{e}(x)\right\}$ has at least $n$ elements below $g(n)$.

Proof. (1) implies (2): Suppose $A$ is r.e. traceable via the recursive function $p(n)$, and let $f \leq_{T} A$. Let

$$
q(n)=\max \{r(i, e, n) \mid i<p(n), e<n\},
$$

where $r(i, e, n)$ is primitive recursive such that

$$
\varphi_{r(i, e, n)}(r(i, e, n)) \simeq \text { the } i \text {-th member of } W_{\varphi_{e}(n)} \text { in order of enumeration. }
$$

Since $f \leq_{T} A$ and $q$ is recursive, $f \circ q \leq_{T} A$, so let $g(n)$ be recursive such that for all $n, f(q(n)) \in W_{g(n)}$ and $\left|W_{g(n)}\right| \leq p(n)$. Let $e$ be an index of $g$, i.e., $\varphi_{e}(n)=g(n)$ for all $n$. 
Suppose for all $n>e$ we have $f(q(n)) \neq \varphi_{r(i, e, n)}(r(i, e, n)) \simeq$ the $i$-th member of $W_{\varphi_{e}(n)}=W_{g(n)}$, for all $i<p(n)$. Since $\left|W_{g(n)}\right| \leq p(n)$, it follows that $f(q(n)) \notin$ $W_{g(n)}$, a contradiction. So $f(q(n))$ must have been equal to $\varphi_{r(i, e, n)}(r(i, e, n))$ for some $i<p(n)$ and all $n>e$. But this gives a bounding function $z(n)$ witnessing that $f$ is not SDNR. This is now easily translated into a bounding function witnessing that any function recursive in $A$ is not even DNR, via the proof of Theorem 2.5.

(2) implies (1): Given $f \leq_{T} A$, let $\hat{f}(x)=(f(0), \ldots, f(x))$. By assumption $\hat{f}(x)=\varphi_{x}(x)$ for $y+1$ many $x$ below $z(y+1)$. Hence there is such an $x$ with $x \geq y$, and so

$$
f(y) \in T_{y}:=\left\{\left(\varphi_{x}(x)\right)_{y}: x \geq y, x<z(y+1)\right\},
$$

for almost every $y$. So by modifying the trace finitely, it holds for every $y$. The size of $T_{y}$ is bounded by $z(y+1)$, since $T_{y}$ contains at most one number for each $x<z(y+1)$.

(3) implies (4): The above argument gives the conclusion that no recursively traceable set computes a function that agrees with each recursive function only finitely often. Indeed, if $W_{g(n)}$ has recursive size, then we can bound the running time of $\varphi_{r(i, e, n)}$ to produce a partial recursive function with recursive domain, which hence has a total recursive extension.

(4) implies (3): This follows the proof that (2) implies (1). If $(f(0), \ldots, f(x))=$ $\varphi_{e}(x)$, then

$$
f(y) \in T_{y}:=\left\{\left(\varphi_{e}(x)\right)_{y}: x \geq y, x<g(y+1)\right\},
$$

for almost every $y$, and the size of $T_{y}$ is bounded by $g(y+1)$.

\section{ACKNOWLEDGMENTS}

The authors would like to thank Cristian S. Calude and André Nies for correspondence and helpful comments to improve the proof of Theorem 4.3. Stephen G. Simpson for the idea to use SDNR functions to express the proof of Theorem 6.2 and A. Khodyrev, who pointed out that one can give an easier proof of the equivalence of complex and autocomplex with DNR by going via SDNR functions.

\section{REFERENCES}

[1] Klaus Ambos-Spies, Bjørn Kjos-Hanssen, Steffen Lempp, and Theodore A. Slaman, Comparing DNR and WWKL, J. Symbolic Logic 69 (2004), no. 4, 1089-1104. MR2135656 (2006c:03061)

[2] Richard Beigel, Harry Buhrman, Peter Fejer, Lance Fortnow, Piotr Grabowski, Luc Longpré, Andrej Muchnik, Frank Stephan, and Leen Torenvliet, Enumerations of the Kolmogorov function, J. Symbolic Logic 71 (2006), no. 2, 501-528. MR2225891 (2007b:68086)

[3] Cristian S. Calude, 2005. Private communication.

[4] Noam Greenberg and Joseph S. Miller, Lowness for Kurtz randomness, J. Symbolic Logic 74 (2009), no. 2, 665-678. MR 2518817 (2010b:03050)

[5] Carl G. Jockusch Jr., Degrees of functions with no fixed points, Logic, methodology and philosophy of science, VIII (Moscow, 1987), Stud. Logic Found. Math., vol. 126, NorthHolland, Amsterdam, 1989, pp. 191-201. MR.1034562 (91c:03036)

[6] M. I. Kanovič, The complexity of the enumeration and solvability of predicates, Dokl. Akad. Nauk SSSR 190 (1970), 23-26 (Russian). MR0262084 (41:6694)

[7] _ The complexity of the reduction of algorithms, Dokl. Akad. Nauk SSSR 186 (1969), 1008-1009 (Russian). MR.0262082 (41:6692)

[8] Bjørn Kjos-Hanssen, André Nies, and Frank Stephan, Lowness for the class of Schnorr random reals, SIAM J. Comput. 35 (2005), no. 3, 647-657 (electronic), DOI 10.1137/S0097539704446323. MR2201451 (2006j:68051) 
[9] Ming Li and Paul Vitányi, An introduction to Kolmogorov complexity and its applications, 2nd ed., Graduate Texts in Computer Science, Springer-Verlag, New York, 1997. MR.1438307 (97k:68086)

[10] , An introduction to Kolmogorov complexity and its applications, 3rd ed., Texts in Computer Science, Springer, New York, 2008. MR 2494387 (2010c:68058)

[11] Joseph Stephen Miller, Pi-0-1 classes in computable analysis and topology, 2002. Ph.D. thesis, Cornell University.

[12] André Nies, 2004. Private communication.

[13] Frank Stephan and Liang Yu, Lowness for weakly 1-generic and Kurtz-random, Theory and applications of models of computation, Lecture Notes in Comput. Sci., vol. 3959, Springer, Berlin, 2006, pp. 756-764. MR2279138 (2007h:03073)

Department of Mathematics, University of Hawail at Mānoa, 2565 McCarthy Mall, Honolulu, HaWAiI 96822

E-mail address: bjoern@math.hawaii.edu

Institut für Informatik, Ruprecht-Karls-Universität HeidelberG, Im Neuenheimer Feld 294, 69120 Heidelberg, Germany

E-mail address: merkle@math.uni-heidelberg.de

Departments of Computer Science and Mathematics, National University of Singapore, 3 Science Drive 2, Singapore 117543, Republic of Singapore

E-mail address: fstephan@comp.nus.edu.sg 